\title{
Laboratório de estudos e práticas em saúde da mulher: relato de experiência
}

\author{
Laboratory of studies and practices in women's health: experience report \\ Laboratorio de estudios y prácticas en salud de la mujer: un informe de experiência
}

Recebido: 12/01/2021 | Revisado: 21/01/2021 | Aceito: 22/01/2021 | Publicado: 30/01/2021

\author{
Graciela Dutra Sehnem \\ ORCID: https://orcid.org/0000-0003-4536-824X \\ Universidade Federal de Santa Maria, Brasil \\ E-mail: graci_dutra@yahoo.com.br \\ Anahy da Silva Machado \\ ORCID: https://orcid.org/0000-0001-7890-0787 \\ Universidade Federal de Santa Maria, Brasil \\ E-mail: anahymachado1@gmail.com \\ Amanda Suélen Monteiro \\ ORCID: https://orcid.org/0000-0002-4170-4501 \\ Universidade Federal de Santa Maria, Brasil \\ E-mail: amandasuelenmonteiro@ hotmail.com \\ Aline Cammarano Ribeiro \\ ORCID: https://orcid.org/0000-0003-3575-2555 \\ Universidade Federal de Santa Maria, Brasil \\ E-mail: alinecammarano@gmail.com \\ Nathalia Kaspary Boff \\ ORCID: https://orcid.org/0000-0003-2709-2438 \\ Universidade Federal de Santa Maria, Brasil \\ E-mail: nathaliakasparyboff@gmail.com \\ Fernanda Duarte Siqueira \\ ORCID: https://orcid.org/0000-0002-1157-1779 \\ Universidade Federal de Santa Maria, Brasil \\ E-mail: fesiqueiraenf@gmail.com \\ Mariana Ferreira Scopel \\ ORCID: https://orcid.org/0000-0001-5867-8840 \\ Universidade Federal de Santa Maria, Brasil \\ E-mail: maryana.scopel@gmail.com \\ Giovana Sangiogo Dallabrida \\ ORCID: https://orcid.org/0000-0001-8383-100X \\ Universidade Federal de Santa Maria, Brasil \\ E-mail: giovana.dallabrida@ hotmail.com \\ Lívia Martins de Martins \\ ORCID: https://orcid.org/0000-0002-6057-217X \\ Universidade Federal de Santa Maria, Brasil \\ E-mail: liviamartinsm13@gmail.com \\ Victória de Quadros Severo Maciel \\ ORCID: https://orcid.org/0000-0003-1959-7639 \\ Universidade Federal de Santa Maria, Brasil \\ E-mail: victoriatrabalhos@outlook.com \\ Eliane Tatsch Neves \\ ORCID: https://orcid.org/0000-0002-559-9533 \\ Universidade Federal de Santa Maria, Brasil \\ E-mail: eliane.neves@ufsm.br
}

\section{Resumo}

Objetivo: Relatar a experiência de desenvolvimento de um projeto de ensino denominado Laboratório de estudos e práticas em saúde da mulher. Métodos: Trata-se de um relato de experiência que descreve as vivências a partir da inserção em atividades no processo de ensino da disciplina Enfermagem na Saúde da Mulher e Pediátrica na Atenção Básica do Curso de Enfermagem de uma universidade pública do sul do Brasil. Os encontros no laboratório ocorreram quinzenalmente, no período de agosto a dezembro de 2019. Relato de experiência: Para simular a prática assistencial foram desenvolvidas simulações realísticas baseadas em casos clínicos relacionados à saúde da mulher e construídos roteiros para nortear as consultas de enfermagem. Ao final, os alunos destacaram pontos negativos e positivos da experiência, discutindo e refletindo sobre o que poderia ser modificado e aperfeiçoado na prática realizada. A vivência de ensino-aprendizagem no laboratório de habilidades, por meio do projeto de ensino, mostrouse positiva para discentes e docentes envolvidos. Essa metodologia propiciou momentos de estudo descontraídos e passíveis de reflexão e aprendizado. Conclusão: Projetos como esse promovem a tomada de decisões, o 
fortalecimento de habilidades e o exercício da liderança, possibilitando maior confiança para desenvolver habilidades e competências essenciais no trabalho do enfermeiro.

Palavras-chave: Treinamento por simulação; Saúde da mulher; Educação em enfermagem; Enfermagem.

\begin{abstract}
Objective: Relate an experience of developing a teaching project called the Laboratory of Studies and Practices in Women's Health. Methods: This is an experience report that describes the experiences from the insertion in activities in the teaching process of the discipline Nursing in Women's Health and Pediatric in Primary Care of the Nursing Course at a public university in southern Brazil. The meetings in the laboratory took place fortnightly, from August to December 2019. Results: To simulate care practice, realistic simulations were developed based on clinical cases related to women's health and roadmaps were built to guide nursing consultations. At the end, the students highlighted negative and positive points of the experience, discussing and reflecting on what could be modified and improved in the practice performed. The teaching-learning experience in the skills laboratory, through the teaching project, proved to be positive for students and teachers involved. This methodology provided moments of study that were relaxed, capable of reflection, and learning. Conclusion: Projects like this promote decision making, the strengthening of skills and the exercise of leadership, enabling greater confidence to develop essential skills and competencies in the work of nurses.
\end{abstract}

Keywords: Simulation training; Women's health; Nursing education; Nursing.

\title{
Resumen
}

Objetivo: Reporte la experiencia de desarrollar un proyecto de enseñanza llamado Laboratorio de Estudios y Prácticas en Salud de la Mujer. Métodos: Este es un informe de experiencia que describe las experiencias de la inserción en actividades en el proceso de enseñanza de la disciplina Enfermería en Salud de la Mujer y Pediatría en Atención Primaria del Curso de Enfermería en una universidad pública en el sur de Brasil. Las reuniones en el laboratorio tuvieron lugar quincenalmente, de agosto a diciembre de 2019. Resultados: Para simular la práctica asistencial, se desarrollaron simulaciones realistas basadas en casos clínicos relacionados con la salud de la mujer y se crearon hojas de ruta para guiar las consultas de enfermería. Al final, los estudiantes destacaron los puntos negativos y positivos de la experiencia, discutiendo y reflexionando sobre lo que podría modificarse y mejorarse en la práctica realizada. La experiencia de enseñanza-aprendizaje en el laboratorio de habilidades, a través del proyecto de enseñanza, demostró ser positiva para los estudiantes y maestros involucrados. Esta metodología proporcionó momentos de estudio relajados y capaces de reflexión y aprendizaje. Conclusión: Proyectos como este promueven la toma de decisiones, el fortalecimiento de habilidades y el ejercicio de liderazgo, permitiendo una mayor confianza para desarrollar habilidades y competencias esenciales en el trabajo de las enfermeras.

Palabras clave: Entrenamiento de simulación; La salud de la mujer; Educación en enfermería; Enfermería.

\section{Introdução}

Nos anos 80 com a criação do Sistema Único de Saúde (SUS), o Ministério da Saúde (MS) elaborou o Programa de Assistência Integral à Saúde da Mulher (PAISM). Esse programa apresentava como princípios norteadores o direito à saúde, à equidade de gêneros, a descentralização e a regionalização dos serviços de assistência. Por meio do PAISM a atenção a saúde da mulher proporcionou maior conhecimento e autonomia sobre sua mente e seu corpo. Já, em 2004, foi elaborada a Política Nacional de Atenção Integral à Saúde da Mulher (PNAISM) com o propósito de melhorar e qualificar a promoção da saúde da mulher em questões delicadas, como, situações de aborto, violência doméstica, planejamento familiar entre outras situações (Barros, Aquino, \& Souza, 2019).

Apesar dos inegáveis avanços, a heterogeneidade brasileira produz e mantém diferenças marcantes nos indicadores entre regiões, estados e municípios (Melo, Aquino, Soares, \& Bevilacqua, 2017). No que tange a mortalidade materna, esta integra o campo da saúde, dos direitos sexuais e reprodutivos das mulheres, representando um evento marcador da qualidade da assistência obstétrica. Neste contexto, de modo a reduzir a morbimortalidade materna, na atenção básica à saúde os profissionais precisam estar atentos e capacitados para acolher, precocemente, a gestante e a família, a fim de identificar e prevenir o surgimento de problemas e dificuldades relacionados à vivência deste período (Vargas et al., 2016).

Já, no que se refere a agravos à saúde da mulher, o câncer de colo de útero tem merecido destaque em muitos estudos devido à sua alta frequência e por ser reconhecidamente uma neoplasia passível de prevenção (Ribeiro et al., 2016; Silveira et al., 2016). Uma redução de $80 \%$ da mortalidade pode ser alcançada por meio do rastreamento para a detecção da doença entre 
mulheres assintomáticas (Ribeiro et al., 2016). Ainda, dentre os agravos, o câncer de mama é um problema de saúde pública no Brasil e um desafio que vem representando para o sistema de saúde no sentido de se garantir o acesso pleno e equilibrado da população ao diagnóstico e tratamento desta doença (Zapponi, Tocantins, \& Vargens, 2015).

Dessa forma, percebe-se a importância de qualificar a formação acadêmica de profissionais da saúde para desenvolverem uma assistência qualificada e segura, contribuindo para o decréscimo desses índices. No ensino, o debate sobre a segurança do paciente desenvolve-se a partir de duas perspectivas, uma que busca garantir que os estudantes desenvolvam as competências necessárias para assistir aos pacientes de modo seguro, e outra que espera que o processo de ensino e aprendizagem dos estudantes em formação não exponha os pacientes a riscos desnecessários. Neste contexto, a simulação clínica é uma estratégia pedagógica orientada pela aprendizagem experiencial que atende ambas as necessidades aproximando estudantes e profissionais aos contextos reais simulados, oferecendo completa segurança para os envolvidos (Oliveira, Massaroli, Martini, \& Rodrigues et al, 2018).

Para tanto, no ensino de graduação, projetos de monitoria desenvolvidos em laboratórios, que possibilitem mimetizar situações clínicas reais, permitem ao estudante de enfermagem o desenvolvimento de habilidades e de competências clínicas atitudinais e psicomotoras (Valadares \& Magro, 2014). A implementação da simulação possibilita aos estudantes praticar e corrigir seus erros frente a situações do cotidiano clínico, sem riscos para o paciente e com o mínimo de risco para si mesmos. A simulação faculta a melhora do desempenho do estudante a partir de seus próprios erros, aprendendo a partir das falhas até o momento em que ocorra o acerto (Valadares \& Magro, 2014).

Para a aplicação das simulações no ensino, podem ser utilizados manequins (simulador de paciente), pessoas no papel de paciente (paciente simulado), objetos virtuais de aprendizagem (softwares educativos) e dramatização (Ribeiro, Garbuio, Zamariolli, Eduardo, \& Carvalho, 2018; Negri et al., 2017). Na construção dos cenários simulados são empregados recursos físicos e materiais que aproximam, com alto grau de realismo, as atividades reais das práticas clínicas com pacientes. Os recursos são definidos de acordo com os objetivos de aprendizagem e são classificados conforme seus potenciais tecnológicos (Negri et al., 2017).

A experiência clínica simulada favorece a consciência clínica e o desenvolvimento de competências de manejo clínico avançado, incluindo habilidades de liderança e de trabalho em equipe (Ribeiro et al., 2018). Ademais, evidências demonstram que os ganhos obtidos com essa tecnologia são variados, entre os quais se destacam a satisfação, autoconfiança, conhecimento, empatia, realismo, diminuição do nível de ansiedade, conforto, comunicação e motivação (Negri et al., 2017).

Justifica-se este relato devido a importância do desenvolvimento de projetos de ensino que possibilitem aos estudantes aprimorarem conteúdos teóricos e desenvolverem simulações realísticas em ambientes protegidos. A relevância na utilização de simulações realísticas se revela na promoção do desenvolvimento de aprendizagens significativas, demonstrando eficácia na educação cognitiva e comportamental dos estudantes (Magnago et al., 2019).

Sendo assim, devido a relevância da temática e a necessidade de aprofundar os conhecimentos acerca da saúda da mulher, tem-se como objetivo relatar a experiência de desenvolvimento de um projeto de ensino denominado laboratório de estudos e práticas em saúde da mulher.

\section{Metodologia}

Trata-se de um estudo qualitativo do tipo relato de experiência, que descreve as vivências no desenvolvimento de atividades do projeto de ensino intitulado: Laboratório de estudos e práticas em saúde da mulher. Este projeto está vinculado a disciplina Enfermagem na Saúde da Mulher e Pediátrica na Atenção Básica, alocada no quarto semestre da graduação, e desenvolvida no Laboratório de Habilidades do Curso de Graduação em Enfermagem da Universidade Federal de Santa Maria. Os encontros foram realizados de agosto a dezembro de 2019 com periodicidade quinzenal. 
Inicialmente, foi realizada a aproximação da bolsista monitora com a temática do projeto a fim de compreender os seus objetivos e metodologia. A bolsista levantou as principais necessidades existentes na turma e referidas pelos alunos. Posteriormente, realizou-se o planejamento dos encontros, implementação de atividades que aliassem teoria à prática e, por fï, avaliação por meio de feedbacks dos discentes e da monitora. Além disso, para que as atividades fossem desenvolvidas e a monitora conseguisse realizar uma assistência de qualidade foi desenvolvida orientação e acompanhamento dos docentes em todo o processo.

Para dar complementaridade ao ensino teórico da disciplina, foram utilizadas Metodologias Ativas de Ensino (MAE). Estas objetivam desenvolver as potencialidades dos acadêmicos para que possam assumir papéis protagonistas no processo de formação, além de estimular a participação frequente no processo dinâmico de construção do conhecimento, de resolução e avaliação de problemas (Hermida, Barbosa, \& Heidemann, 2015).

No que se refere às simulações realísticas, seguiram-se as seguintes etapas (Jeffries, Rodgers \& Adamson, 2015). No primeiro momento ocorreu a definição dos temas a serem trabalhados, etapa na qual abordaram-se questões referentes ao prénatal, puerpério e prevenção de câncer de colo de útero e mama. As referências bibliográficas que embasaram tais conteúdos foram enviadas para a turma via e-mail. Após, na segunda etapa, definiram-se os objetivos de cada simulação. Neste momento, objetivava-se propiciar um ambiente de uma cena semelhante ao atendimento à saúde da mulher em uma unidade de atenção primária à saúde. Após, na terceira etapa, foram elaborados casos clínicos, roteiros de execução e de avaliação. No quarto momento, organizou-se o cenário. Posteriormente, na quinta fase, os discentes assumiram papeis de atores da cena. E, por fim, realizou-se a discussão após a simulação.

O laboratório de habilidades era previamente organizado de maneira a tornar aquele espaço o mais próximo da realidade das unidades de atenção primária à saúde com serviços de atenção à saúde da mulher, objetivando estimular os alunos a desenvolverem as atividades, bem como reconhecerem suas dificuldades, potencialidades e a importância daquela prática assistencial. Com isso, tinham a oportunidade de avaliar as próprias condutas, juntamente com seus erros e acertos e, assim, aprender e construir novas habilidades.

Para ilustrar as situações e aproximar da realidade, fez-se uso dos bonecos anatômicos disponíveis no laboratório, os quais configuraram-se como uma excelente ferramenta no processo de observação realizada pela monitora e docente sobre as fragilidades e potencialidades dos discentes em cada etapa desenvolvida.

Ao final de cada encontro, houve a realização de feedbacks pelos discentes sobre as suas percepções dos papéis desenvolvidos e sugestões para próximos encontro bem como da monitora e docente. Essa ferramenta revelou-se importante para a formação do aluno (Kaneko \& Lopes, 2019), pois a partir de comentários dos envolvidos no cenário e de como se sentiram desempenhando tais atividades, foi possível ajustar a metodologia adotada no processo de ensino-aprendizagem.

\section{Resultados}

Como cenário para a simulação realística (SR) foi utilizado o laboratório de habilidades do Curso de Enfermagem. A bolsista monitora contou com auxílio de uma enfermeira Técnica Administrativa em Educação (TAE) alocada no Curso de Enfermagem para organizar o ambiente com os equipamentos necessários para a simulação, quais sejam: bonecos anatômicos, sonar, fita métrica, espátula ayre, escova endocervical, espéculo, entre outros.

No primeiro momento era explicado o que seria feito, além da construção de um roteiro para consulta ginecológica e de pré-natal, sendo oportunizado um momento para retirada de dúvidas a respeito do instrumento elaborado. A partir disso, iniciava-se a simulação realística utilizando casos clínicos, nos quais a bolsista atuou no papel de paciente fictícia, respondendo aos questionamentos e relatando seus sintomas e características previamente estabelecidos nos casos clínicos, e os discentes como enfermeiros. Enquanto um acadêmico realizava a simulação, os demais estudantes do grupo observavam e faziam 
anotações complementares em seus roteiros pessoais. Ao final, a bolsista solicitava um feedback dos alunos espectadores da simulação, proporcionando uma primorosa discussão dos pontos positivos e negativos.

$\mathrm{Na}$ simulação de consulta ginecológica, por exemplo, os estudantes precisavam atentar para o breve histórico apresentado pela monitora que atuava como paciente, realizar orientações a partir das falas da paciente e de seu parceiro, desenvolver a técnica correta do exame clínico das mamas e a coleta do citopatológico em modelos anatômicos. A simulação exigia diversos conhecimentos para uma assistência de qualidade, tendo em vista a necessidade de priorizar a escuta ativa da paciente, analisar e orientar quanto à promoção da sua saúde sexual e reprodutiva e averiguar comportamentos de risco para desenvolver câncer de colo de útero ou mama.

A bolsista organizou, juntamente com a docente, casos clínicos que contemplavam diferentes situações de mulheres atendidas em um serviço de atenção primária à saúde. Esta ferramenta foi utilizada com o intuito de os acadêmicos identificarem sinais e sintomas de câncer de colo de útero e mama, presença de infecções sexualmente transmissíveis (IST) e as peculiaridades da mulher nas fases da adolescência e do climatério. Para cada etapa da consulta realizada, era esperado que o estudante atentasse para os sinais e sintomas que pudessem indicar possíveis alterações e que realizasse questionamentos, orientações e condutas adequadas e imprescindíveis à resolução de cada caso.

Em uma das simulações realísticas de consulta ginecológica, o acadêmico apresentava conhecimento acerca do exame clínico das mamas e de como orientar o autoexame para a paciente. Entretanto, este não considerou questões de sexualidade, como, por exemplo, a informação de que a paciente no climatério apresentava dispareunia devido à falta de lubrificação, não a orientando de como poderia proceder para suavizar o desconforto sentido durante as relações sexuais. Já outro acadêmico, não realizou orientações à paciente acerca da importância do uso de preservativo e de como realizar a higiene íntima de forma correta, da mesma forma que ao coletar o material para o exame citopatológico não a informou sobre como este exame é realizado. Com isso, a partir da discussão realizada após simulação, foi possível identificar as fragilidades nas condutas clínicas dos discentes e rever as possibilidades para aqueles atendimentos.

Já a segunda simulação tratava da consulta de pré-natal de risco habitual em uma unidade de atenção primária à saúde. Os casos clínicos embasaram-se na identificação de gestação de risco habitual e de alto risco, nesta última contemplou-se o diabetes gestacional, a pré-eclâmpsia e a eclâmpsia. A situação contemplava o acolhimento das gestantes, a anamnese, a reavaliação de risco gestacional, o cálculo da idade gestacional, a solicitação e avaliação de exames laboratoriais e de imagem, a avaliação do peso, a verificação da pressão arterial (PA), a técnica da manobra de Leopold Zweifel, a medida da altura uterina, a ausculta de batimentos cardíacos fetal (BCF), as orientações pertinentes a cada trimestre, os encaminhamentos necessários e o agendamento de consulta de retorno. Os acadêmicos deveriam, nestas situações, atentar para as queixas das gestantes e sinais e sintomas que indicassem fatores de risco, além de desenvolverem competências técnicas da consulta de pré-natal.

Durante a simulação realística de um dos casos clínicos, um discente calculou corretamente a idade gestacional da paciente e realizou orientações quanto às vacinas que deveriam ser realizadas, porém não solicitou os exames laboratoriais. Também, não identificou sinais e sintomas que evidenciavam alterações na gestação. Em outro momento, um discente apresentou insegurança na realização da medida da altura uterina, mas identificou a necessidade de suplementação com sulfato ferroso a partir da $20^{\mathrm{a}}$ semana gestacional.

Os acadêmicos, no momento de discussão, reconheceram que suas falhas na simulação se fossem cometidas na prática assistencial poderiam causar danos à mulher ou ao feto, aumento os riscos de morbimortalidade materna e neonatal. A partir dessas experiências, relataram que se sentiam mais seguros para iniciar as aulas práticas da disciplina, pois tinham sanado suas dúvidas, além de identificarem os pontos que necessitavam de maior aprofundamento teórico. Além disso, relataram sentiremse sentiram após terem seus os pontos positivos exaltados e fortalecidos. Com isso, o conteúdo aprendido em aula teórica pode 
reforçado durante a simulação, oportunizando aos acadêmicos a possibilidade de rever suas condutas e melhorá-las após ter suas fragilidades identificadas e discutidas.

Foi possível observar, também, que os acadêmicos aderiram e mostraram mais interesse nas atividades da monitoria, previamente, ao início das atividades práticas, devido à insegurança de atuar frente a uma situação real. Ademais, sugeriram a ampliação das atividades também para a área da saúde da criança no contexto da atenção primária à saúde. Ainda, expressaram que, por se tratar de uma monitora também acadêmica, sentiram-se mais à vontade para perguntar e esclarecer dúvidas.

Após o término das simulações, os acadêmicos atuantes em cena e os observadores puderam verbalizar quanto às condutas corretas e as que deixaram a desejar e o que fariam no lugar do colega para prestar melhor assistência à gestante, permitindo uma reflexão sobre a experiência. Com isso, oportunizou-se um espaço para elencar os pontos negativos e os positivos da consulta realizada durante a simulação, assim como as informações que foram esquecidas e/ou que deveriam ser reforçadas para as atividades práticas. Já, ao final do encontro, a bolsista deu seu feedback quanto aos pontos que deveriam ser estudados para melhorar a atuação na consulta, fazendo com que os acadêmicos conseguissem visualizar a importância do conhecimento teórico prévio para melhor desenvolvimento da prática e, consequentemente, para aprimorar o cuidado realizado em sua trajetória acadêmica.

\section{Discussão}

Percebe-se a necessidade de profissionais da área da saúde com postura humanizada, crítica e reflexiva, pautada em conhecimento teórico e científico. Para isso, as instituições de ensino também precisaram sofrer modificações no processo de ensino-aprendizagem para formar profissionais que atendam ao perfil esperado. Entretanto, ainda existem dificuldades para tornar esta prática mais condizente com a realidade profissional, uma vez que o conteúdo disciplinar é centrado em aprender por técnicas de memorização tradicionais de ensino (Silva et al., 2015). Neste sentido, para que o ensino em saúde incorpore os avanços pedagógicos de maneira a estabelecer novas e atualizadas diretrizes para aliar teoria à prática, é necessário adotar MAE que promovam uma formação além do perfil básico e clínico (Roman et al., 2017).

No processo de ensino e formação de enfermeiros, as MAE permitem uma conexão entre a teoria, os serviços de atenção à saúde e a população assistida, tendo em vista a autonomia dos acadêmicos na construção de seus conhecimentos, leitura da realidade assistencial e trabalho em equipe (Hermida et al., 2015). Além disso, as MAE propõem uma mudança de paradigma onde o aluno não fica somente absorvendo o conhecimento que lhe é transmitido pelo professor que é visto como detentor do conhecimento no modelo tradicional de ensino, neste caso o aluno é responsabilizado pelo seu próprio aprendizado e para alcança-lo necessita ir em busca de conhecimento se tornando o protagonista do processo (Pereira, et al., 2018).

A utilização de MAE ainda estimula a curiosidade e mantém o interesse dos alunos fazendo com que tracem metas para adquirir conhecimento. De forma a tornar os alunos profissionais com pensamento crítico-reflexivo quanto a qualidade da sua prática profissional para com os usuários (Silva, et al., 2020).

Com a explicação de como seriam os encontros de monitoria, os discentes demonstraram maior entusiasmo por terem a oportunidade de praticar as consultas de enfermagem de forma segura, em ambiente protegido, a partir de possíveis casos reais apresentados. Desse modo, a monitoria constitui uma ferramenta de apoio pedagógico por meio da qual o discentemonitor e o assistido têm oportunidade de aprofundar conhecimentos, fortalecer habilidades teórico-práticas e esclarecer dúvidas, sanando fragilidades inerentes a uma área de conhecimento (Andrade, Rodrigues, Nogueira, \& Souza, 2018).

Para tornar o processo de ensino-aprendizagem mais dinâmico e ativo, optou-se por trabalhar com simulações realísticas, a partir das quais foi possível observar o comprometimento e a evolução dos discentes ao demonstrarem o uso do conhecimento teórico, o tempo de resposta para os problemas levantados e o planejamento de estratégias e tomadas de decisões. Esse gerenciamento de atitudes, permite visualizar o comportamento dos futuros profissionais ao desenvolverem 
essas e outras práticas assistenciais, bem como o respeito à relação com os indivíduos assistidos e a postura para lidar com os desafios de trabalhar em equipe (Magnago et al., 2019).

A percepção dos discentes sobre ter o monitor como um facilitador da aprendizagem, consolida uma troca aberta de conhecimentos e aprimoramento de habilidades (Araújo, Pires, \& dos Santos, 2019). Essas experiências podem ser justificadas devido à equidade de categorias entre monitor e monitorados, uma vez que ambos se encontram no contexto de aprendizagem (Araújo et al., 2019).

A utilização da simulação realística auxiliou no imaginário dos discentes na resolução dos casos clínicos, pois ao representar o papel de profissional enfermeiro, eles deveriam observar todos as situações clínicas de determinado caso e, assim, planejar a assistência necessária para a resolução dos problemas descritos. Desde modo, esta estratégia facultou o raciocínio clínico e o pensamento crítico, possibilitando uma prática segura, minimizando os riscos e aprimorando a atuação do estudante frente ao paciente, familiares e equipe de saúde.

Pelo custo razoável e grande possibilidade de aplicação, o uso de práticas simuladas com recursos da dramatização pode se tornar excelente aliado para o aprimoramento de profissionais com pensamento crítico e reflexivo, capazes de realizar julgamento clínico e tomada de decisão. A dramatização pode ser definida como uma representação teatral determinada a partir de um foco ou tema. É um recurso que possibilita ao aprendiz integrar teoria e prática, é flexível e ajustável a vários contextos, permite experiências de diferentes perspectivas e pontos de vista, assim como oferece ao aprendiz a oportunidade de explorar a vulnerabilidade individual em ambiente seguro (Negri et al., 2017).

Ressalta-se que a aprendizagem alicerçada na simulação tem sinergias claras com o currículo do curso de Graduação em Enfermagem e apresenta consistência com a intenção educacional da atualidade. Esta ferramenta enriquece o processo de ensino-aprendizagem, sendo uma estratégia de ensino relevante e que pode ser implementada na grade curricular, pelo potencial de ampliar as competências e habilidades do estudante. Além disso, possibilita uma maior aproximação dos discentes entre si, aumentando a desenvoltura e a confiança deles durante a realização das aulas práticas. Ademais, percebe-se que projetos como esse promovem a busca pelo conhecimento, propiciando a tomada de decisões e o exercício da liderança do enfermeiro. Tais atributos são competências essenciais para atuação deste profissional frente às situações clínicas comuns ao cotidiano da prática assistencial do enfermeiro no cuidado à saúde da mulher.

\section{Considerações Finais}

A vivência de ensino-aprendizagem no laboratório de habilidades do Curso de Graduação em Enfermagem, por meio do desenvolvimento do projeto de ensino Laboratório de estudos e práticas em saúde da mulher, mostrou-se positiva para os discentes e também para os docentes envolvidos. Essa metodologia propiciou um momento de estudo descontraído e passível de reflexão e aprendizado. Além disso, por meio do exercício da resolução dos casos clínicos e a utilização de simuladores percebeu-se maior motivação dos discentes na busca do conhecimento e aprofundamento de conteúdos abordados em sala de aula.

Entende-se que este relato traz, também, importantes contribuições para a reflexão quanto à importância e efetividade da monitoria acadêmica no processo ensino-aprendizagem e sua relevância no contexto da educação superior. No que tange às limitações, entende-se que parte das percepções e inferências aqui discutidas podem não ser passíveis de generalização, dada a influência de fatores acadêmico-científicos, socioculturais, econômicos e organizacionais. Mas, certamente, podem contribuir para reflexão e adaptação em outras realidades semelhantes, como as demais universidades públicas, por exemplo.

Para além disto, expectamos com o desenvolvimento deste estudo que o mesmo incite a produção científica a experenciar, estudar e aprimorar as metodologias ativas no processo de ensino e aprendizagem no âmbito dos laboratórios de enfermagem e também para além destes, levando em consideração os resultado promissores encontrados na aplicação desta 
metodologia no processo de formação de acadêmicos de enfermagem.

\section{Referências}

Andrade, E. G. R., Rodrigues, I. L. A., Nogueira, L. M. V., \& Souza, D. F. (2018). Contribuição da tutoria acadêmica para o processo de ensino-aprendizagem na graduação em Enfermagem. Revista Brasileira de Enfermagem, 71 (Suppl. 4): 1596-1603.

Araújo, M., Pires, P., \& dos Santos, E. (2019). Monitoria como atividade de ensino-aprendizagem sob a ótica de acadêmicos de enfermagem. Revista Enfermagem Atual In Derme, 89(27).

Barros, P. de S., Aquino, E C. de, \& Souza, M. R. de (2019). Mortalidade fetal e os desafios para a atenção à saúde da mulher no Brasil. Rev. Saúde Pública, 53(31): 240-252.

Hermida, P. M. V., Barbosa, S. S., \& Heidemann, I. T. S. B. (2015). Metodologia ativa de ensino na formação do enfermeiro: inovação na atenção básica. Revista de Enfermagem da UFSM, 5(4): 683-691.

Jeffries, P. R., Rodgers, B., Adamson, K (2015). NLN Jeffries Simulation Theory: Brief Narrative Description. Nurs Educ Perspect., v. 36, n. 5, p. 292-3.

Kaneko, R. M. U, \& Lopes, M. H. B. M. (2019). Cenário em simulação realística em saúde: o que é relevante para a sua elaboração? Revista da Escola de Enfermagem da USP, 53 (e03453).

Magnago, T. S. B. S., Silva, J. S. da Lanes, T. C., Ongaro, J. D., Luz, E. M. F. da, Tuchtenhagen, P., \& Andolhe, R. (2019). Simulação realística no ensino de segurança do paciente: relato de experiência. Revista de Enfermagem da UFSM, 10(13): 1-16.

Melo, C. M. de, Aquino, T. I. S., Soares, M. Q., \& Bevilacqua, P. D. (2017). Vigilância do óbito como indicador da qualidade da atenção à saúde da mulher e da criança. Ciência \& Saúde Coletiva, 22(10): 3457-3465.

Negri, E. C., Mazzo, A., Martins, J. C. A., Pereira Junior, G. A., Almeida, R. G. dos S., \& Pedersoli, C. E. (2017). Clinical simulation with dramatization: gains perceived by students and health professionals. Revista Latino-Americana de Enfermagem, 25: e2916.

Oliveira, S. N. de Massaroli, A., Martini, J. G., \& Rodrigues, J. (2018). Da teoria à prática, operando a simulação clínica no ensino de Enfermagem. Revista Brasileira de Enfermagem, 71(Suppl. 4): 1791-1798.

Pereira, A. S., Shitsuka, D. M., Parreira, F. J., Shitsuka, R. (2018). Metodologia da pesquisa científica (ed. 1). Metodologias Ativas (pp. 54-62). Santa Maria: Universidade Federal de Santa Maria.

Ribeiro, L., Bastos, R. R., Vieira, M. de T., Ribeiro, L. C., Teixeira, M. T. B., \& Leite, I. C. G. (2016). Rastreamento oportunístico versus perdas de oportunidade: não realização do exame de Papanicolaou entre mulheres que frequentaram o pré-natal. Cadernos de Saúde Pública, $32(6)$ : e00001415.

Ribeiro, V. dos S., Garbuio, D. C., Zamariolli, C. M., Eduardo, A. H. A., \& Carvalho, E. C. de. (2018). Simulação clínica e treinamento para as Práticas Avançadas de Enfermagem: revisão integrativa. Acta Paulista de Enfermagem, 31(6): 659-666.

Roman, C., Ellwanger, J., Becker, G., Da Silveira, A., Machado, C., \& Manfroi, W. (2017). Metodologias ativas de ensino-aprendizagem no processo de ensino em saúde no Brasil: uma revisão narrativa. Clinical \& Biomedical Research, 37(4).

Silva, R.P., Camacho, A. C. L. F., Silva, M. A. P. da \& Menezes, H. F. de. (2020). Estratégias de utilização da metodologia ativa na formação de acadêmicos de enfermagem: relato de experiência. Revista Pesquisa, Sociedade e Desenvolvimento, [S. 1.], v. 9, n. 6, pág. e160963543, 2020.

Silva, S. L. da, Silva, S. F. R. da, Santana, G. S. de M., Nuto, S. de A. S., Machado, M. de F. A. S., Diniz, R. de C. M., \& Sá, H. L. do C. (2015). Estratégia Educacional Baseada em Problemas para Grandes Grupos: Relato de Experiência. Revista Brasileira de Educação Médica, 39 (4): $607-613$.

Silveira, N. S. P., Vasconcelos, C. T. M., Nicolau, A. I. O., Oriá, M. O. B., Pinheiro, P. N. da. C., \& Pinheiro, A. K. B. (2016). Conhecimento, atitude e prática sobre o exame colpocitológico e sua relação com a idade feminina. Revista Latino-Americana de Enfermagem, 24 : e2699.

Valadares, A. F. M., \& Magro, M. C. (2014). Opinião dos estudantes de enfermagem sobre a simulação realística e o estágio curricular em cenário hospitalar. Acta Paulista de Enfermagem, 27(2): 138-143.

Vargas, A. S., Alves, V. H., Rodrigues, D. P., Branco, M. B. L. R., Souza, R. de M. P. de, \& Guerra, J. V. V. (2016). Atuação dos profissionais de saúde da estratégia saúde da família: promoção da prática do aleitamento materno. Revista Baiana de Enfermagem, 30(2): 1-9.

Zapponi, A. L. B., Tocantins, F. R., \& Vargens, O. M. da. (2015). O enfermeiro na detecção precoce do câncer de mama e no âmbito da atenção primária. Revista Enfermagem UERJ, 23(1): 33-38. 